\title{
A NEW CONFIGURATION FOR A DIPOLE MAGNET FOR USE IN HIGH ENERGY PHYSICS APPLICATIONS*
}

\author{
D. I. MEYER and R. FLASCK
}

Physics Department, University of Michigan, Ann Arbor, Michigan 48104, U.S.A.

Received 16 December 1969

The magnetic field configuration obtained when an ordinary circular solenoid is skewed looks very promising for use as a dipole magnet. The field inside is uniform and $\int B \mathrm{~d} l$ including end effect is independent of position across the magnet.

At the high fields now attainable with superconducting materials the support of the long narrow coils which are needed in bending magnets for high energies becomes a problem. In addition considerable rather difficult coil placement must be done to attain uniform fields. I suggest here a configuration which eliminates both of these problems.

Consider a long circular solenoid skewed at an angle $\theta$ as shown in fig. 1 . On analysis of the field pattern it is found that the field inside such a configuration is completely uniform and makes an angle of $\theta / 2$ with the $z^{\prime}$ axis. Details of this analysis are given in the appendix. The magnitude of the $z^{\prime}$ component of the magnetic field is the same as that of an unskewed solenoid with the same current and turn spacing in the $z$ direction.

If we now send a beam of particles through along the skewed axis they will be bent perpendicular to the plane of the paper by the component $B_{x^{\prime}}$ of the field. Thus the effective bending will be $B \sin \frac{1}{2} \theta$. We may however improve on this configuration by the method illustrated

* Work performed under the sponsorship of the U.S. Atomic Energy Commission.

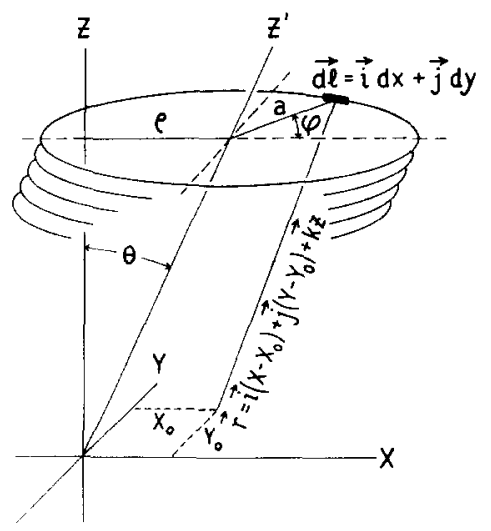

Fig. 1. Diagram for calculating the magnetic field at the point $x_{0}, y_{0}$. in fig. 2. Place two skewed solenoid windings on top of each other but skewed in opposite direction. Then by applying currents as shown the $z^{\prime}$ field components cancel but the $x^{\prime}$ components add. Thus we have ellipsoidal cross sections with a completely uniform field perpendicular to the axis in the $x^{\prime}$ direction.

Clearly since we are cancelling one field component there is some effective loss of ampere turns. Approximately twice as much wire is needed as in a standard cosine dipole for a given field. However for a superconducting magnet this is not too serious and is compensated for by four factors.

1. The field is completely uniform and the $\int B \cdot \mathrm{d} l$ through the magnet including end effects is independent of lateral position.

2. The field volume is used very effectively. The aperture viewed along the beam line is elliptical with the long axis of the ellipse in the direction of bend. For $60^{\circ}$ skew for example the aperture for $10 \mathrm{~cm}$ diameter rings would be $10 \mathrm{~cm}$ in the direction of bend and $5 \mathrm{~cm}$ perpendicular to this direction.

A uniform field of elliptic cross section can be generated by the proper distribution of current $\left(J \propto y^{\prime} / a\right)$ in wires run along the $x^{\prime}$ direction however the end effects are not so neatly handled.

3. The mechanical construction is much simplified over that of a cosine dipole from the standpoint of properly placing the wires. The circular coils and compact structure simplify the handling of magnetic stresses.

4. The configuration lends itself very well to being made in modules which can then be stacked end to end to provide any desired bending. This could add

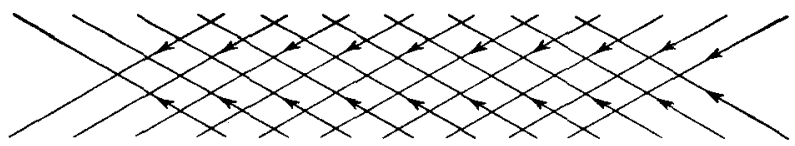

Fig. 2. Two superimposed coils with opposite skew. 
greatly to the flexibility of setting up beams and minimize the variety of magnets of different lengths which would otherwise be needed.

The end effects were studied by using a computer sum of individual loops. Fig. 3 shows a curve on axis with a skew angle of $60^{\circ}$. A four layer coil having radii of $1,1 \frac{1}{3}, 2 \frac{2}{3}$ and 3 skewed left, right, right and left respectively was used. The amount of overshoot and undershoot depends on the skew angle, and lateral position inside the solenoid. Fig. 4 shows dependence on $x^{\prime}$ and $y^{\prime}$ position in the solenoid. All numbers are in units of coil radii. Notice that the overshoot and undershoot in fig. 3 are symmetric. A simple symmetry argument shows why this symmetry must occur and why therefore the $\int B_{x^{\prime}} \mathrm{d} z^{\prime}$ must be independent of position across the solenoid even though the exact shape of the field differs from point to point as shown in fig. 4 . Consider two infinite coils with the same radii skewed in opposite directions. Now think of them as split in two halves shown dashed and solid in fig. 5 . The field in the coil must be constant along $z^{\prime}$ if we take the sum of that due to the two halves. Therefore, where the field due to the solid half overshoots, that due to the dashed half must go negative by exactly the same amount. Now consider two points A and B placed symmetrically to the split in the coil but otherwise arbitrary. The $B_{x}$ field component at $A$ due to the solid coil (dashed coil) must be the same as that at $B$ due to the dashed coil (solid coil) by symmetry. Thus the amount of undershoot at B due to the solid coil

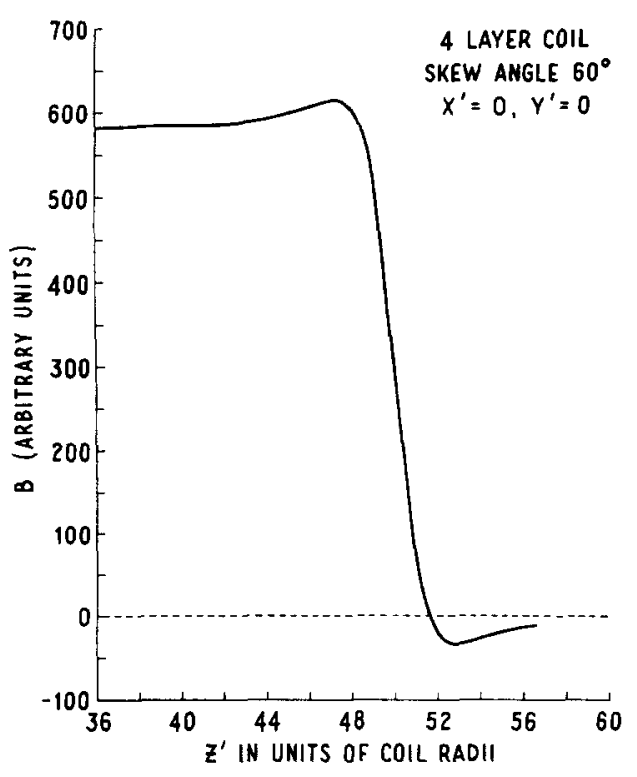

Fig. 3. End effect of magnet on center line. must equal the overshoot at $\mathrm{A}$ due to the solid coil. This same argument can be used for $x^{\prime}=0$ even for a single coil or for many coils of different radii. For $x^{\prime}=0$ the symmetry argument fails if the coils of different skew have different radii. However by alternating the skew of the coil layers the $\int B_{x^{\prime}} \mathrm{d} z^{\prime}$ is constant over the entire solenoid area to a very high order.

A model was made using 4 coils, the innermost being $10 \mathrm{~cm}$ in diameter. These were skewed at $70^{\circ}$ angles on an elliptic tube. Field measurements show a field uniformity across the aperture (centered in the $z^{\prime}$ direction) better than $1 \%$. (This was the limit of accuracy of the measurements.) They also verified the end effects which we calculated. No great care was taken in making a true elliptical shape. In places the deviation from a true ellipse was as large as $1 \mathrm{~mm}$. Also no great care was taken to accurately set the skew angle of all coils the same. Thus as might be expected mechanical tolerances do not seem very critical.

Because of the uniformity of the field and of the end effects, magnets with this design should be valuable not only in beam lines but also as spectrometer magnets and in storage rings.

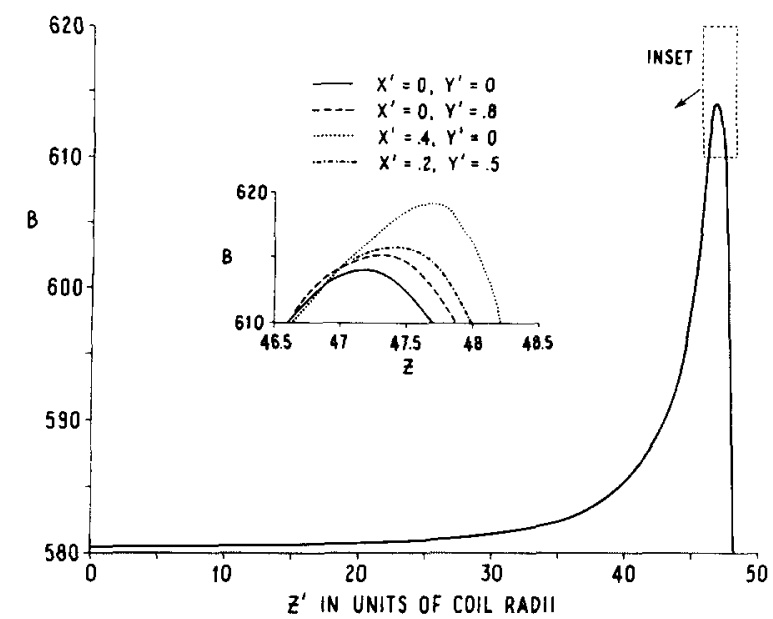

Fig. 4. Detail of overshoot in fig. 3 as a function of position inside coil.

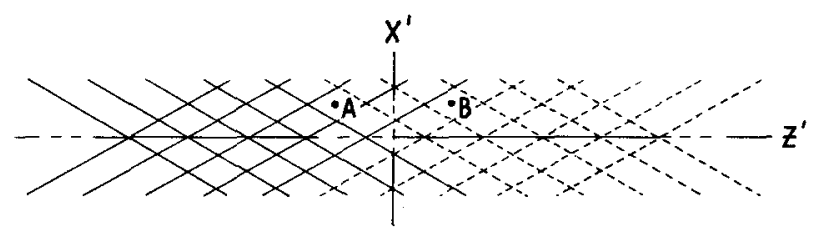

Fig. 5. The symmetry of two coils joined together. 
We whould like to thank Dr. L. W. Jones for useful discussions during the course of this work.

\section{Appendix}

It is convenient in analyzing the field of a skewed solenoid to set up two coordinate systems as shown in fig. 1, one with $z$ perpendicular to the planes of the individual coils, one with $z^{\prime}$ along the solenoid axis. If $l$ is the current in each turn of the coil it is simple to find $B_{z^{\prime}}$, from Ampere's law. Since

$$
\oint B \cdot \mathrm{d} i=\mu_{0} I
$$

we get

$$
B_{z^{\prime}}=\mu_{0} I n^{\prime}=\mu_{0} I n \cos \theta
$$

Here $n^{\prime}$ is the number of turns per unit length along $z^{\prime}$, $n$ the number per unit length along $z$. Notice that $B_{z}{ }^{\prime}$ is independent of position within the solenoid.

To find other components of the field in the solenoid is more difficult. By reference to fig. 1 we can set up the equation for a small length $\mathrm{d} l$ of wire in a particular loop as

$\mathrm{d} B=\frac{\mu_{0} I}{4 \pi} \frac{[\boldsymbol{i} \mathrm{d} x+\boldsymbol{j} \mathrm{d} y] \times\left[\boldsymbol{i}\left(x-x_{0}\right)+\boldsymbol{j}\left(y-y_{0}\right)+\boldsymbol{k}(z)\right]}{\left[\left(x-x_{0}\right)^{2}+\left(y-y_{0}\right)^{2}+\left(z^{2}\right)\right]^{\frac{3}{2}}}$.

One can then pick the $x$ component of the field and write the equation in terms of polar coordinates. This gives

$$
\mathrm{d} B_{x}=\frac{\mu_{0} I}{4 \pi} \frac{z a \cos \varphi \mathrm{d} \varphi}{\left[\left(a \cos \varphi+\rho-x_{0}\right)^{2}+\left(a \sin \varphi-y_{0}\right)^{2}+z^{2}\right]^{\frac{3}{2}}} .
$$

If an attempt is made to integrate this expression over $\varphi$ one finds it to be an elliptic integral. For a finite solenoid which must be studied to understand and effects the procedure has been to evaluate these elliptic integrals then add them together for the appropriate coil spacing, skew angle, etc., by means of a computer.

For an infinitely long solenoid an analytic solution can be found. Note that

$$
\begin{aligned}
& x=\rho+a \cos \varphi, \\
& y=a \sin \varphi, \\
& \rho=z \tan \theta .
\end{aligned}
$$

Then replace the individual turns by an equivalent current per unit length $J=I n$ and integrate over $\mathrm{d} z$ from $-\infty$ to $+\infty$. This integral when evaluated gives $\mathrm{d} B_{x}=\frac{\mu_{0} J}{2 \pi} \sin \theta \cos ^{2} \theta$.

$$
\left[\frac{a \cos \varphi\left(a \cos \varphi-x_{0}\right) \mathrm{d} \varphi}{\cos ^{2} \theta\left(a \cos \varphi-x_{0}\right)^{2}+\left(a \sin \varphi-y_{0}\right)^{2}}\right] .
$$

One must still integrate over $\varphi$ but now the elliptic integral expression no longer appears. The expression to be integrated is not a standard form that can be found in integral tables. A hint as to how to proceed comes from the fact that we do not expect $B_{x}$ to have a simple dependence outside the solenoid. Thus it is probable that the resultant integrated expression should be applicable only inside the solenoid region. Note that the denominator becomes 0 for some value of $\varphi$ when $\left(x_{0}^{2}+y_{0}^{2}\right) / a^{2}=1$. A contour integral is indicated the solution to which is applicable only inside the solenoid. Therefore substitute $\delta=\mathrm{e}^{\mathrm{i} \varphi}$ in the expression for $\mathrm{d} B_{x}$ above. The integral of $\varphi$ from 0 to $2 \pi$ then becomes an integral around a contour $C$ of unit radius

$B_{x}=\frac{\mu_{0} J}{2 \pi} \sin \theta \cos ^{2} \theta$.

$\iint_{C} \frac{\left(\delta^{2}+1\right)\left(\delta^{2}-2 \frac{x_{0}}{a} \delta+1\right) \mathrm{d} \delta}{\left.\cos ^{2} \theta\left(\delta^{2}-2 \frac{x_{0}}{a} \delta+1\right)^{2}+\left(-\mathrm{i} \delta^{2}-\frac{2 y_{0}}{a} \delta+\mathrm{i}\right)^{2}\right]}$

This expression has simple poles within the contour at $\delta=0$ and

$$
\delta=\frac{\frac{x_{0} \cos \theta}{a}-\mathrm{i} \frac{y_{0}}{a} \pm\left[\left(\frac{x_{0}}{a} \cos \theta-\mathrm{i} \frac{y_{0}}{a}\right)^{2}+\sin ^{2} \theta\right]^{\frac{1}{2}}}{\cos \theta+1} .
$$

The evaluation of the residues of these poles proceeds in a straightforward but tedious manner. Taking the sum of the residues gives

$$
B_{x}=\mu_{0} J \cos \theta \tan \frac{1}{2} \theta=\mu_{0} i^{\prime} \tan \frac{1}{2} \theta .
$$

Thus $B_{x}$ is independent of position inside the solenoid and is equal to $B_{z}^{\prime} / \tan \frac{1}{2} \theta$. A little trigonometry then shows that

$$
B_{x}=B_{x^{\prime}}, B_{z}=B_{z^{\prime}} .
$$

The field in the solenoid is uniform and makes an angle of $0 / 2$ with the solenoid axis. A computer sum of the elliptic integrals of individual circular loops gives the same result. By the curl and divergence theorems $B_{y}=B_{y^{\prime}}=0$. 